\title{
AC/DC Current Transformer with Single Winding
}

\author{
P. Ripka ${ }^{1}, K$. Draxler $^{1}$, and R.Styblikova ${ }^{2}$ \\ ${ }^{1}$ Czech Technical University, Prague, Czech Republic, \\ ${ }^{2}$ Czech Metrology Institute, Prague, Czech Republic
}

\begin{abstract}
DC currents in power grids are created by induction from geomagnetic variations and newly also by transformerless power inverters installed in solar power stations. These currents may cause serious errors of current transformers (CTs) measuring the consumed energy, but also cause overload of distribution transformers which may result in blackout. Installing DC current sensors into the grid would be very expensive. We have shown that the existing CTs in the grid can be used in the fluxgate mode to simultaneously measure DC current. Utilizing this mode, the CT AC error is inevitably increased. We analyze the sources of these errors and show techniques to reduce them. The experiments were made on the off-the-shelf $500 \mathrm{~A} / 5 \mathrm{~A}$ CT. We show that the uncompensated 5A DC current without compensation increases its AC amplitude error from $0.1 \%$ to $1 \%$. We also found that the dominant additional error was caused by the increased burden. With the optimized design of the excitation circuit it is possible to sense a DC current component with $10 \%$ accuracy and reduce the additional AC error from previously reported $1.5 \%$ to $0.1 \%$ when the DC current component is compensated. This technique can be widely used to improve the AC accuracy of the existing CTs.
\end{abstract}

Index Terms-current sensors, instrument current transformer, fluxgate, measurement of DC current component

\section{INTRODUCTION}

$\mathrm{F}$ UXGATE DC current sensors have superior DC offset stability compared to current sensors based on Hall effect, but these devices are complicated, expensive and power consuming $[1,2]$. They have usually 2 ring cores excited in opposite directions. Besides the conductor with the measured current, fluxgate current sensors have three windings: excitation winding, detection winding and feedback winding for the compensation of the DC current component. The fluxgate sensor described in [3] utilizes an additional core working as a conventional AC current transformer (CT) in order to increase the sensor bandwidth up to $170 \mathrm{kHz}$. Similar sensor for small currents with an optimized core geometry reached accuracy below $0.1 \%$ [4]. Similar sensors are manufactured by LEM, PREMO, and other manufacturers.

In [5] we have shown that this principle can be utilized using ordinary current transformers which are already mounted in existing installations. The AC/DC CT shown in Fig. 1 works simultaneously as the AC CT and the fluxgate DC current sensor. In the conventional CT mode the measured AC current $I_{1 A C}(f)$ (usually at $f=50 \mathrm{~Hz}$ ) is converted to the AC voltage $V_{2}(f)$ across the burden $R_{2}$. In the fluxgate mode the transformer is excited by the current $\mathrm{I}_{\mathrm{ex}}$ at the frequency $\mathrm{f}_{\mathrm{ex}}$ injected into the excitation winding, using the $\mathrm{AC}$ power generator and the transformer TR. The DC current $\mathrm{I}_{1 \mathrm{DC}}$ is then measured through the voltage $\mathrm{V}_{2}\left(2 \mathrm{f}_{\mathrm{ex}}\right)$ at the second harmonics of the excitation frequency. The excitation frequency $f_{e x}$ is usually much higher than the frequency $f$ of the measured current and its harmonics in order to separate these signals from $\mathrm{f}_{\mathrm{ex}}$ which always leaks into the secondary winding.

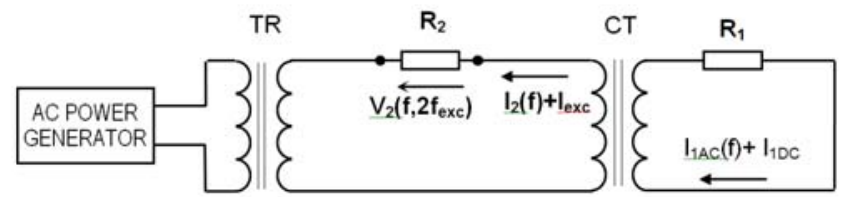

Fig.1: Current transformer CT working in the fluxgate mode. $\mathrm{I}_{2}\left(2 \mathrm{f}_{\text {exc }}\right)$ and $\mathrm{I}_{1}\left(\mathrm{f}_{\text {exc }}, 2 \mathrm{f}_{\text {exc }}\right)$ are not indicated in the figure as they are small, but they are not negligible. For the experiments the AC power generator is replaced by a combination of laboratory function generator and power amplifier.
The significant disadvantage of the AC/DC CT described in [1] was serious degradation of the AC accuracy from $0.1 \%$ error up to $10 \%$ error. This was acceptable for protection purposes, but not in case when the CT is still used for energy measurement.

In this paper we investigate the sources of such additional error and show the ways how to decrease it. We also investigate the effect of the DC current component on the accuracy of $\mathrm{CT}$ and discuss possibilities of reducing this effect by CT design and by compensation. If such sensor is developed it can be widely used in power distribution networks to monitor DC currents caused by the transformerless power inverters and induced by the geomagnetic activity. These currents ranging in extreme cases up to hundreds of Amperes seriously affect the energy measurement and in extreme cases cause the transformer overloads and blackouts.

\section{ACCURACY OF AC CT}

In this section we examine the accuracy of the AC CT outside the nominal conditions but relevant to our application.

The measurements were made on the off-the shelf CT CLA 2.2 with $500 \mathrm{~A} / 5$ A current ratio and nominal burden of $0.2 \Omega$ corresponding to the rated output load of 5VA [5VA is calculated as $\left.0.2 \Omega \cdot(5 \mathrm{~A})^{2}\right]$. For this load the error is below 0.1 $\%$ from $5 \%$ to $120 \%$ of the nominal primary current (FS) of $500 \mathrm{~A}$. The positive amplitude (or ratio) error $\varepsilon_{\mathrm{I}}$ indicates that the manufacturer applied a turn correction to improve the specifications. The amplitude error of the CT itself should be always negative, as the loss angle in the core $\delta$ is always positive and the phase displacement $\beta$ of the resistive secondary burden is close to zero [2]. The amplitude error $\varepsilon_{I}$ of the CT at line frequency can be written as

$$
\varepsilon_{I}=\frac{N_{2}\left|\mathbf{I}_{2}\right|-N_{1}\left|\mathbf{I}_{1}\right|}{N_{1}\left|\mathbf{I}_{1}\right|} 100 \cong-\frac{I_{m} \sin (\delta+\beta)}{I_{1}} 100
$$

First we investigated the influence of the inevitably increased burden. Fig. 2 shows that CT with $0.8 \Omega$ (20VA) burden still meets its $0.2 \%$ specs from $10 \% \mathrm{FS}$. 

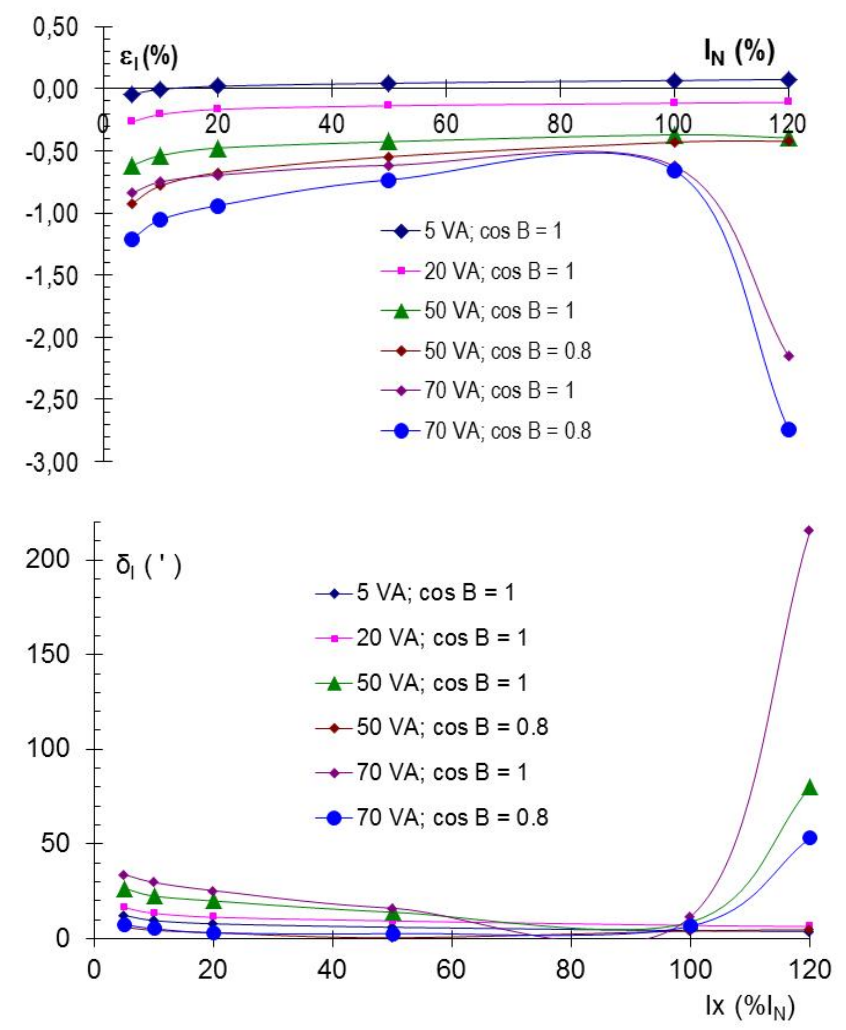

Fig. 2: Amplitude error $\varepsilon_{\mathrm{i}}(\%)$ and phase error $\delta_{\mathrm{i}}\left({ }^{\circ}\right)$ of the CT (for measured currents from $5 \%$ to $120 \%$ of the nominal current range $\mathrm{I}_{\mathrm{N}}$ ) for several values of burden. The power factor $\cos \mathrm{B}$ is either 1 or 0.8 as required by calibration standards.

Fig. 3 shows how the ordinary CT loaded by the nominal burden is sensitive to the DC current. The error increased from below $0.2 \% / 15^{\prime}$ to $1 \% / 130^{\prime}$ for $\mathrm{I}_{1 \mathrm{DC}}$ up to $4.5 \mathrm{~A}$.

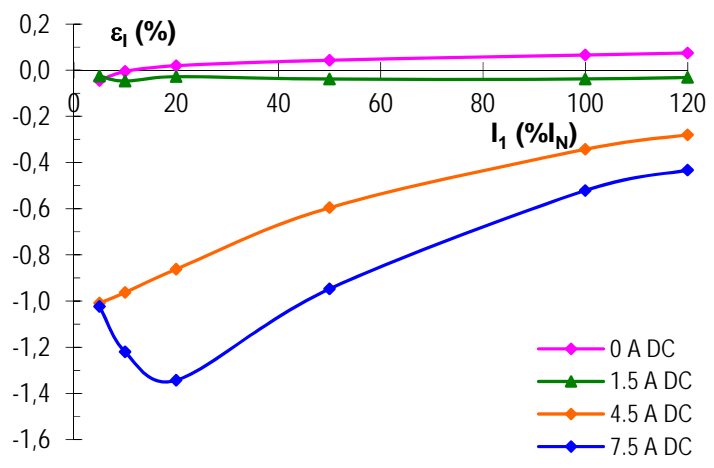

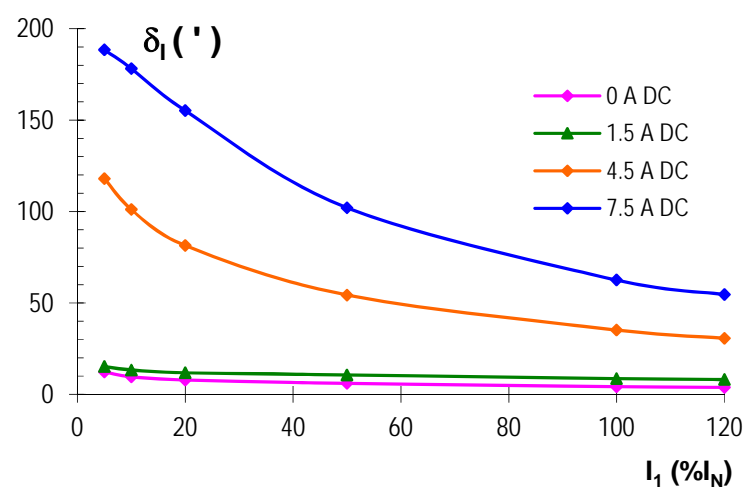

Fig. 3 Amplitude and phase error of the AC CT as a function of $\mathrm{I}_{\mathrm{IDC}}$

DC component in the measured current causes serious degradation of the CT accuracy. This degradation may persist even after the DC current disappears if the core remanence is not demagnetized by a large measured AC current [6]. This effect can be kept low by following measures: - using high $\mathrm{B}_{\mathrm{s}}$ materials for the core (e.g. replacing permalloy by Fe-based nanocrystalline alloy)

- using an airgap in the core or a low-permeability core. This results in the increased basic error which can be corrected numerically or by the electronic circuit such as in [7].

- compensating the DC flux by the current injected into the secondary winding

In order to compensate for it, the DC flux should be measured. We suggest using the fluxgate effect for this purpose. The compensation scheme can be used not only to decrease the error of the $\mathrm{CT}$, but also to control the transformerless gridconnected power converters to reduce the DC current component injected into the grid similarly as [8].

We should point out that a serious DC magnetization of a CT can also be detected by the numerical analysis of the measured data. However, these methods fail for the weak magnetization (typically caused by the core remanence) due to numerical unstability [9]. Some authors suggest measuring the DC voltage across the power line resistance to monitor the DC current [10] - however due to the changes of the grid impedance the sensitivity is unpredictable and this method can only be used as a null indicator in the feedback compensation systems.

\section{DC OPERATION OF THE DC/AC CT}

In order to keep the basic function of the CT, the secondary winding should be short-circuited through a small resistive burden. We achieved this by using the lowimpedance excitation transformer TR and small sensing resistor $\mathrm{R}_{2}$ (Fig. 1). We will show that the secondary impedance of this transformer is critical for the AC accuracy. This impedance can be written as

$$
Z_{2}^{2}=\left[R_{2 C u}+R_{\text {out }}\left(\frac{N_{2}}{N_{1}}\right)^{2}\right]^{2}+\left[Z_{\text {lleak }}\right]^{2}
$$

where $\quad \mathrm{R}_{2 \mathrm{Cu}}$ is the resistance of the secondary winding $R_{\text {out }}$ is the output impedance of the excitation power amplifier 
$\mathrm{N}_{1}$ and $\mathrm{N}_{2}$ are the numbers of turns for the primary a and the secondary windings

$\mathrm{Z}_{2 \text { leak }}$ is the leakage inductance of the secondary winding

$\mathrm{Z}_{\text {2leak }}$ can be made negligible if the magnetic core has a closed shape such as a ring core made of a high permeability material. Large ring diameter and large saturation induction Bs help to keep the ring far from saturation. The resistance $\mathrm{R}_{\text {out }}$ at the excitation frequency can also be made in the order of several $\mathrm{m} \Omega$. In this sense we optimized the design of the excitation transformer since [5] and the dominant for $\mathrm{Z}_{2}$ become $\mathrm{R}_{2 \mathrm{Cu}}$. We used two different secondary windings with $\mathrm{R}_{2 \mathrm{Cu}}=1.25 \Omega$ and $0.5 \Omega$ and compare their performance.

The CT operation does not allow using the classical detection of the second harmonic voltage as we excite the CT from the almost ideal sinewave voltage source. We therefore replaced voltage sensing by sensing 2 nd harmonic component in the excitation current. This was made by the lock-in amplifier. For our experiments we have used a DSP lock-in SR 830 , but the $2^{\text {nd }}$ harmonic detection can be made by a simple integrated circuit such as AD630. The reference signal is derived from the excitation voltage.

Fig. 4 shows the $V_{\text {exc }}$ and $I_{\text {exc }}$ waveforms for several values of the $\mathrm{I}_{\mathrm{DC}}$. The $\mathrm{V}_{\mathrm{exc}}$ is sinewave in each condition. While the $\mathrm{I}_{\mathrm{exc}}$ for $\mathrm{I}_{\mathrm{DC}}=0$ is also sinewave, any measured DC current causes the $2^{\text {nd }}$ harmonic component in the $\mathrm{I}_{\text {exc }}$, which may be large: for $150 \mathrm{~A}$ primary DC current (10 A into 15 Turns) the $2^{\text {nd }}$ harmonic response in the secondary winding is $12 \mathrm{~A}_{\mathrm{p}-\mathrm{p}}$. One can be afraid that such current is transformed into the primary circuit to the 100-times higher current. Fortunately, in this case the transformer operation is far from a current transformer even for a very small primary impedance and the actual current injected into the primary circuit is much smaller, as already discussed in [5].

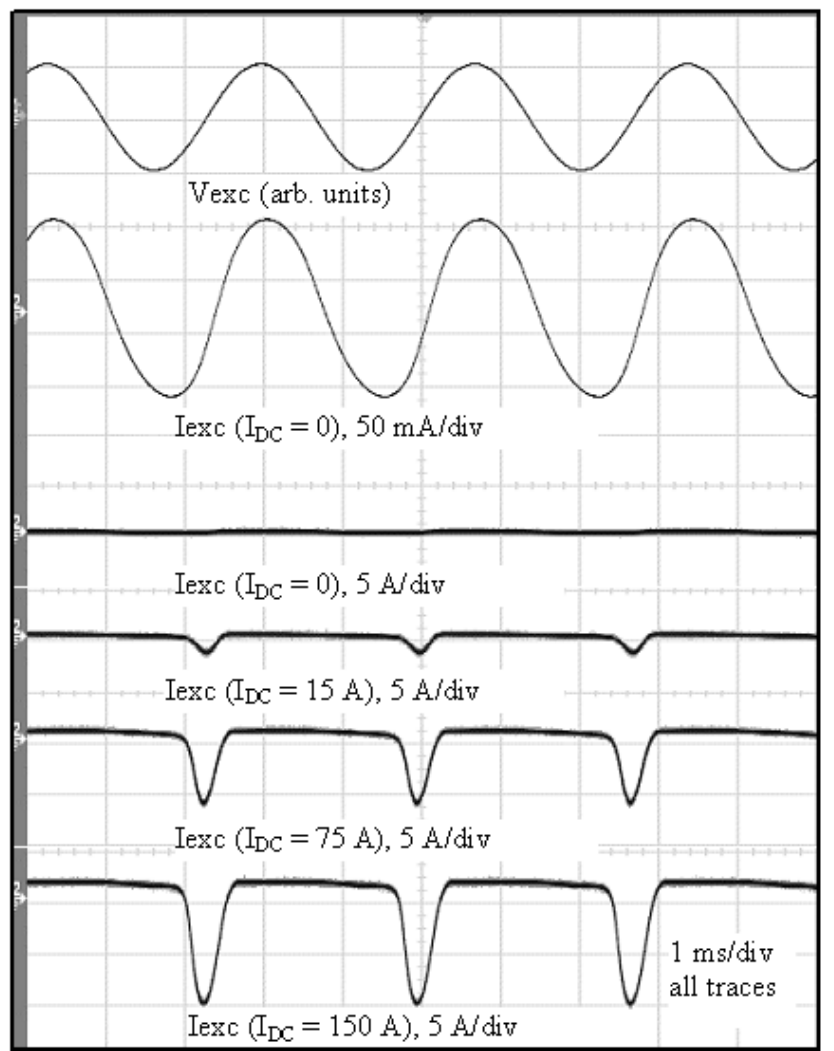

Fig. 4: Waveform of the excitation voltage and excitation current for several values of DC current. Iexc is shown in large magnification in trace 2 with scale $50 \mathrm{~mA} / \mathrm{div}$. The following traces have larger scale $(5 \mathrm{~A} / \mathrm{div})$.

The characteristics of the uncompensated DC/AC current transformer for two values of the excitation voltage and two values of the impedance in the primary circuit is shown in Fig. 5. The weak point of this mode of operation is the influence of impedance in the measured circuit on sensitivity. We have already shown that this can be effectively suppressed below $1 \%$ by stabilizing the excitation voltage instead of the current. In this case the amplitude of the excitation generator was only adjusted for $\mathrm{I}_{\mathrm{DC}}=0$ and kept constant during the measurement. More ideal, but also more difficult to implement would be to stabilize the voltage across the secondary winding of the CT.

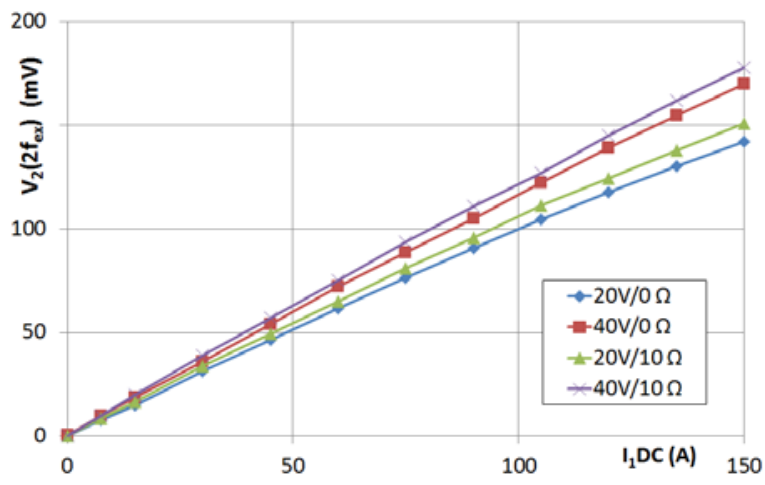

Fig. 5: DC characteristics of the DC/AC CT for $20 \mathrm{~V}$ and $40 \mathrm{~V}$ excitation level and two values of $R_{1}$. This characteristics shows a second harmonic component of voltage across the burden resistor $\mathrm{R}_{2}$. It was measured in 
uncompensated mode.

\section{AC ACCURACY}

CT errors in Figs. 2 and 3 were measured by a Tettex 2767 automatic transformer test set and a Tettex 4764 current comparator. When we applied the $370 \mathrm{~Hz}$ excitation, this electronic measurement system becomes unreliable. We had to return back to basics and built our own measurement setup shown in Fig. 6. Here Tx is the measured CT, while Tn is the standard AC current comparator Tettex 4764. Both transformers are supplied in the primary winding by $\mathrm{AC}$ current source in parallel with adjustable resistor $\mathrm{R}_{1}$, which simulates the influence or the grid resistance. DC component of the measured current is introduced by using auxiliary 15turns winding. DC voltage source is AC decoupled by serial choke. The $370 \mathrm{~Hz}$ excitation is injected through transformer TR.

The secondary currents $\mathrm{I}_{\mathrm{N}}$ and $\mathrm{I}_{\mathrm{A}}$ of $\mathrm{Tn}$ and $\mathrm{Tx}$ are subtracted so that the real and imaginary part of the voltage across $R_{D}$ shows the amplitude and phase errors respectively. These voltage components are measured by the lock-in amplifier. This is possible due to the fact that both CTs behave as the current sources with a very high impedance. The resistor $R_{N}$ serves for the measurement of the current amplitude by voltmeter $\mathrm{V}$.

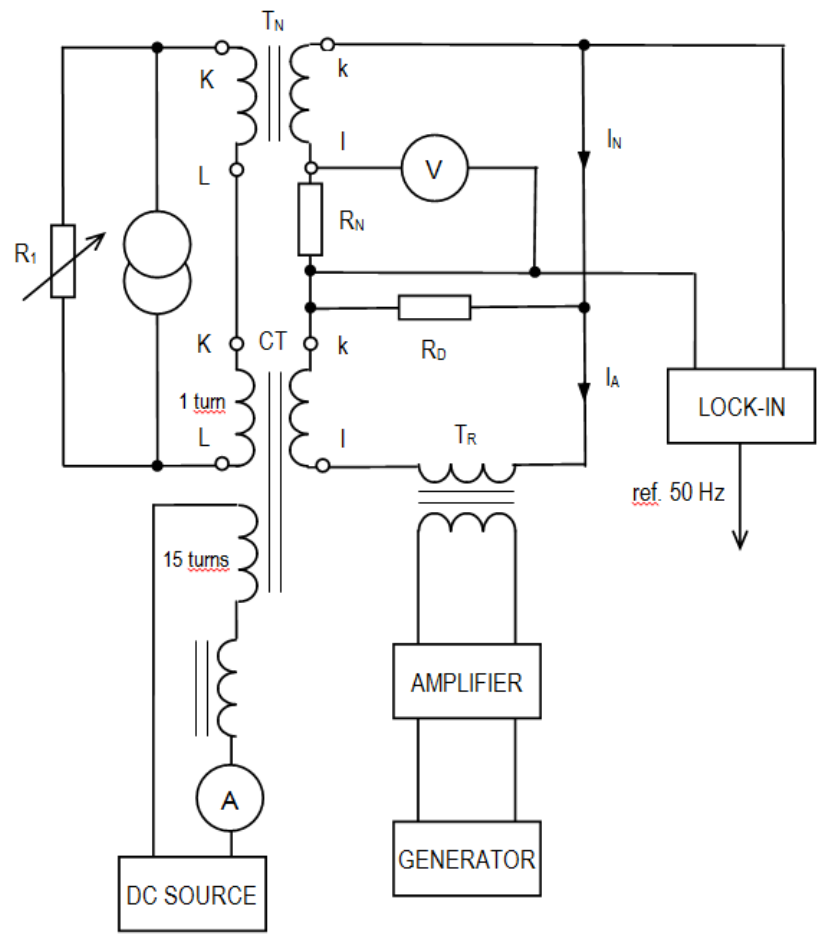

Fig. 6: Measurement setup to evaluate AC errors

First we measured using excitation transformer with 1.25 $\Omega$ output impedance (Fig.7). The increased burden caused additional error at the low measured currents (below $20 \%$ of $\mathrm{I}_{\mathrm{N}}$ ). Adding the $\mathrm{AC}$ excitation actually reduced this error. To our knowledge this effect in CTs was not yet described. It is similar to the magnetic shaking observed in shieldings. Large error for $I_{1}>50 \% I_{N}$ is caused by the saturation. The best results were achieved with $40 \mathrm{~V}$ excitation, where the maximum $\mathrm{AC}$ error was $0.5 \%$.

The measurement error was further reduced by using $0.5 \Omega$ excitation transformer (Fig. 8). The magnetic shaking suppressed the error for small current amplitudes from $0.9 \%$ to $0.05 \%$. The overall error is $0.1 \%$.

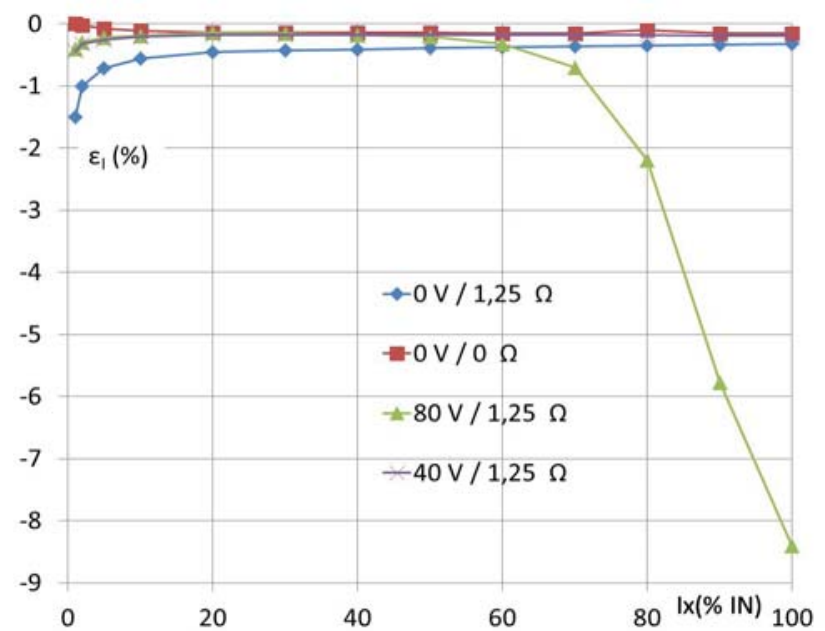

Fig. 7: AC errors of CT for $1.25 \Omega$ excitation transformer

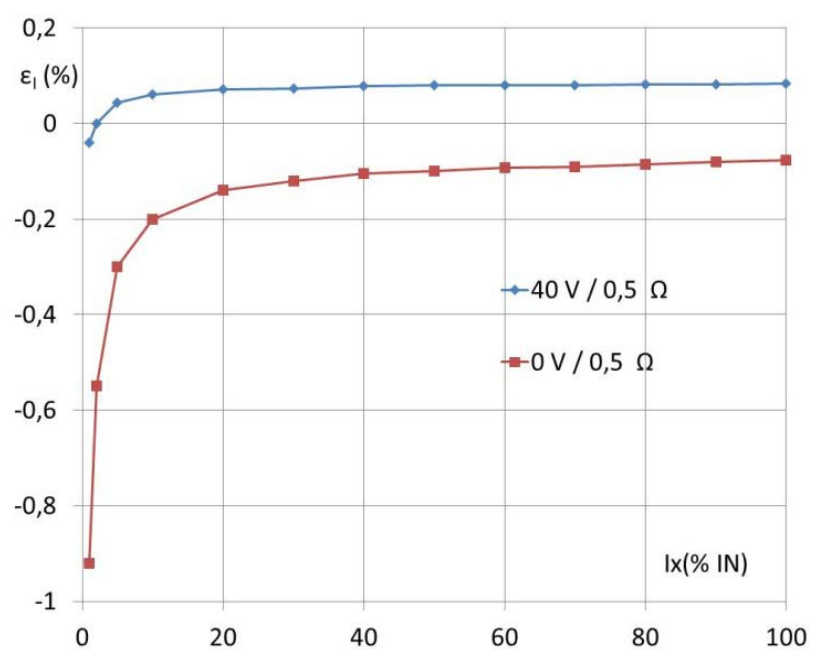

Fig. 8: AC errors of CT for $0.5 \Omega$ excitation transformer

Fig. 9 shows the effect of the uncompensated DC current on the accuracy of the AC current measurement. For DC currents up to $3 \mathrm{~A}$ the error is below $1 \%$. This is comparable to the measured CT in the conventional mode (Fig. 3). Further reduction of this error can be achieved by a DC current compensation. 


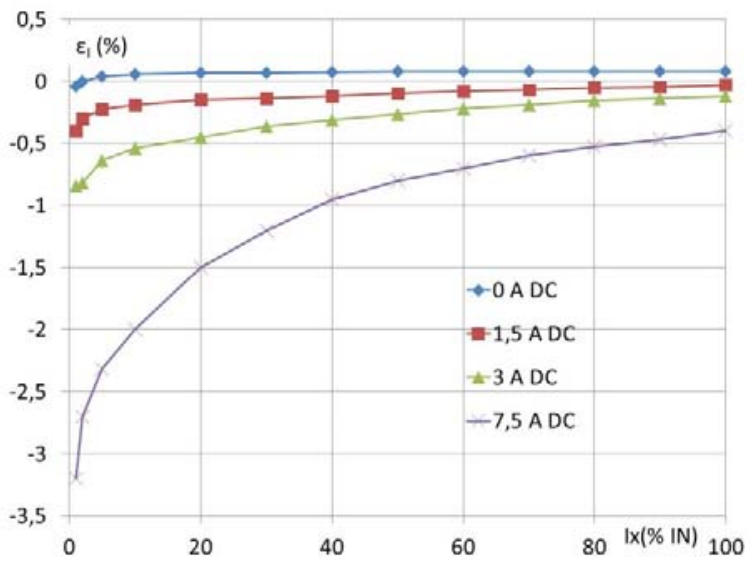

Fig. 9: AC error of DC/AC CT excited by $40 \mathrm{~V} / 370 \mathrm{~Hz}$ for several values of the uncompensated DC current $\mathrm{I}_{1} \mathrm{DC}$.

\section{CONCLUSIONS}

Off-the-shelf CT with a single winding can be converted to the $\mathrm{AC} / \mathrm{DC}$ sensor. Components needed for this are excitation power generator, excitation transformer and lock-in amplifier. No additional winding or software is necessary. By using the low-impedance excitation transformer, the additional error in the $\mathrm{AC}$ mode was reduced from $1.5 \%$ reported in [5] to $0.1 \%$ if the DC current component is not present or manually compensated. In the uncompensated mode the error reaches $1 \%$ for $3 \mathrm{~A} \mathrm{DC}$ and it rapidly increases with increasing DC current level. This type of error is observed also in the conventional CT mode. Our improved sensor opens the way to reduce this type of error without replacing the installed CTs. Possibilities of automatic compensation of the DC component will be investigated in the future work.

\section{ACKNOWLEDGMENT}

This work was partly supported by the Czech Science Foundation grant P102-12-2177.

\section{REFERENCES}

[1] Kaluza, A. Grüger, and H. Grüger, "New and future applications of fluxgate sensors," Sens. Actuators A, Phys., vol. 106, pp. 48-51, 2006.

[2] P. Ripka, Electric current sensors: a review, Meas. Sci. Instrum. 21 (2010) Iss. 11, pp.1-23.

[3] G. Velasco-Quesada, M. Román-Lumbreras, A. Conesa-Roca, and F. Jeréz: Design of a Low-Consumption Fluxgate Transducer for HighCurrent Measurement Applications, IEEE Sen. J., 11, (2010), 280

[4] Yang, Xiaoguang; Zhang, Bo; Wang, Youhua; et al.: The optimization of dual-core closed-loop fluxgate technology in precision current sensor, J. App. Phys. 111 (2012) 07E722

[5] Ripka, P. - Draxler, K. - Styblíková, R.: Measurement of DC currents in the Power Grid by Current Transformer, IEEE Transactions on Magnetics. 2013, vol. 49, no. 1, p. 73-76.

[6] Draxler, K. - Styblíková, R.: Effect of Magnetization on Instrument Transformers Errors In: Journal of Electrical Engineering. 2010, vol. 61 , no. $7 / \mathrm{s}$, p. $50-53$.

[7] N. McNeill, H. Dymond, P. H Mellor: High-Fidelity Low-Cost Electronic Current Sensor for Utility Power Metering, IEEE Trans. on Power Delivery 26 (2011), 2309

[8] Buticchi, G., Lorenzani E., Franceschini G. A DC Offset Current Compensation Strategy in Transformerless Grid-Connected Power Converters, IEEE Trans. Pow. Delivery 26 (2011),pp 2743-2751
[9] Chi-Shan $\mathrm{Yu}$, Detection and Correction of Saturated Current Transformer Measurements Using Decaying DC Components, IEEE Trans. Power Delivery 25 (2010), 1340-1346

[10] Buticchi G., Consolini L., Lorenzani E.: Active Filter for the Removal of the DC Current Component for Single-Phase Power Lines, IEEE Trans. Ind. Electronics 60 (2013),pp 4403-4414. 